\title{
Allergen Skin Response Intensity
}

National Cancer Institute

\section{Source}

National Cancer Institute. Allergen Skin Response Intensity. NCI Thesaurus. Code C112222.

The semiquantitative assessment of the wheal-and-flare index that measures the intensity of the reaction. 